\title{
Primary cerebellopontine angle glioblastoma in a child. A rare entity
}

\author{
Kaviraj Kaushik ${ }^{1}$, Sharad Pandey ${ }^{1}$, L.N. Gupta ${ }^{1}$, \\ Rahul Varshney ${ }^{1}$ \\ 1 Department of Neuro Surgery, P.G.I.M.E.R.D.R Ram Manohar Lohia \\ Hospital, New Delhi, INDIA
}

\begin{abstract}
Cerebellopontine angle extraaxial glioblastoma (GBM) is extremely rare at any age but especially in children. We reported a case of 14-year-old girl, who presented with nausea, vomiting and ataxia. She was evaluated with computed tomography (CT) and magnetic resonance imaging (MRI). Imaging demonstrated irregular ring enhancing right CP angle mass. The atypical findings of irregular ring enhancement, CP angle location and presentation in childhood, combine to make the prospective diagnosis of GBM a difficult one. This combination of findings has been reported very rarely.
\end{abstract}

\section{INTRODUCTION}

Most lesions involving the cerebellopontine angle (CPA) are located extra-axially. The common differentials at this location include acoustic schwannomas, meningiomas, epidermoid tumours, metastases, and arachnoid cysts. Glioblastomas are usually intraxial and mostly in supratentorial compartments and usually in elderly age groups. GBM rarely present in cerebellopontine angle (CPA). Most of cases reported are primary intraaxial GBM arising from cerebellar hemisphere or brainstem, with exophytic extension into CPA. Pubmed advanced search only single of primary extraaxial GBM in the CPA has been reported, arising from the region of root entry zone of the eighth cranial nerve1. Regardless of the site of origin, tumours in the CPA represent with sign and symptoms resulting from compression of fifth, seventh, and eighth cranial nerves and pons and cerebellar peduncle. Because these lesions have different treatment modalities, prognosis, and outcome, so it is important to make differential diagnosis of these lesions. Appropriate diagnosis is important for the management of these lesion, as clinical manifestations of these are similar.

\section{CASE REPORT}

A 14-year-old girl, presented to Neurosurgery OPD with a several week histories of progressively increasing frontal headaches, projectile vomiting and excessive drowsiness and recent onset left sided weakness. Neurological examination revealed GCS-E3V2M6 with left

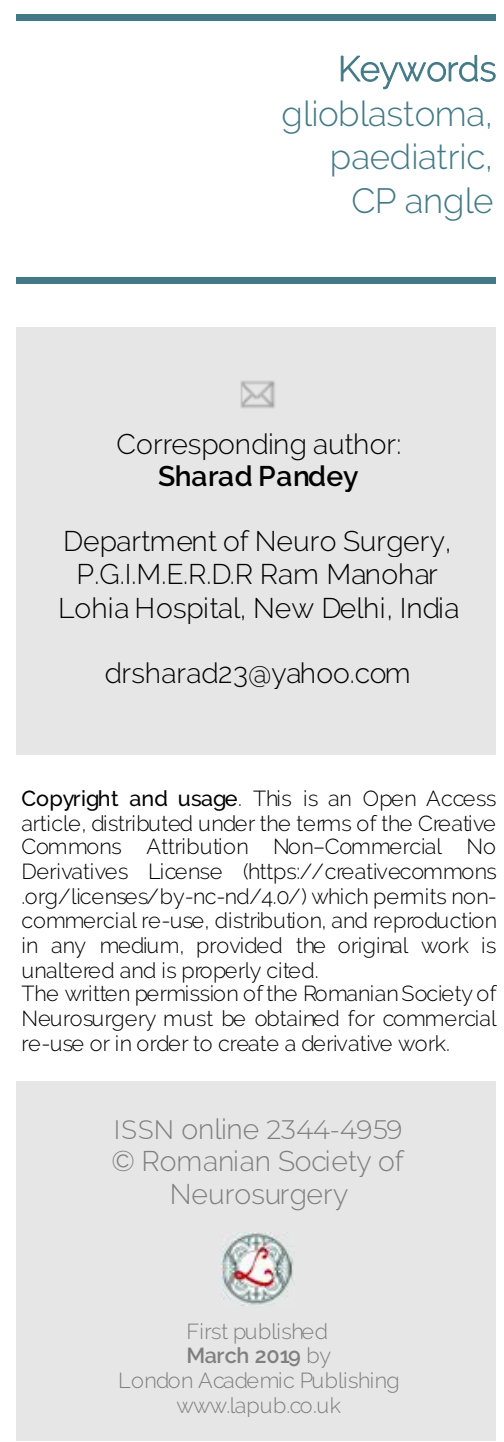


sided hemiparesis and right sided 5, 6, $7^{\text {th }}$ and lower cranial nerve palsies.

Computed tomography (CT) demonstrated right $\mathrm{CP}$ angle mass lesion of size $5 \times 3.5 \mathrm{~cm}$ with effacement of fourth ventricle with obstructive hydrocephalous, with irregular ring enhancement. Magnetic resonance (MR) imaging demonstrated the lesion to be essentially isointense on $\mathrm{T} 1$ and hyperintense on $\mathrm{T} 2$ weighted sequence (Fig.1), and restricted diffusion on DWI. Post contrast T1 weighted images demonstrated irregular peripheral enhancement. There were areas of peripheral blooming on SWI in periphery of mass lesion in pons area (suggestive of hemorrhage). Patient underwent emergent ventriculoperitoneal shunt to relieve hydrocephalus. But patient condition did not improve and further deteriorated after VP shunt and emergency right retromastoid suboccipital craniectomy with tumour decompression was done. Intraoperatively the tumour mass was situated extraxially in $\mathrm{cp}$ angle, extending across $7,8^{\text {th }}$ cranial nerves complex to lower cranial nerves and medially extending to brainstem. Morphologically it was grayish pink, highly vascular, soft and CUSA amenable. Pathologic examination was conclusive for Glioblastoma Multiforme. Patient did not improve neurologically even after tumour decompression and finally expired 2 months after surgery. The diagnosis of GBM could be made after histopathological examination.
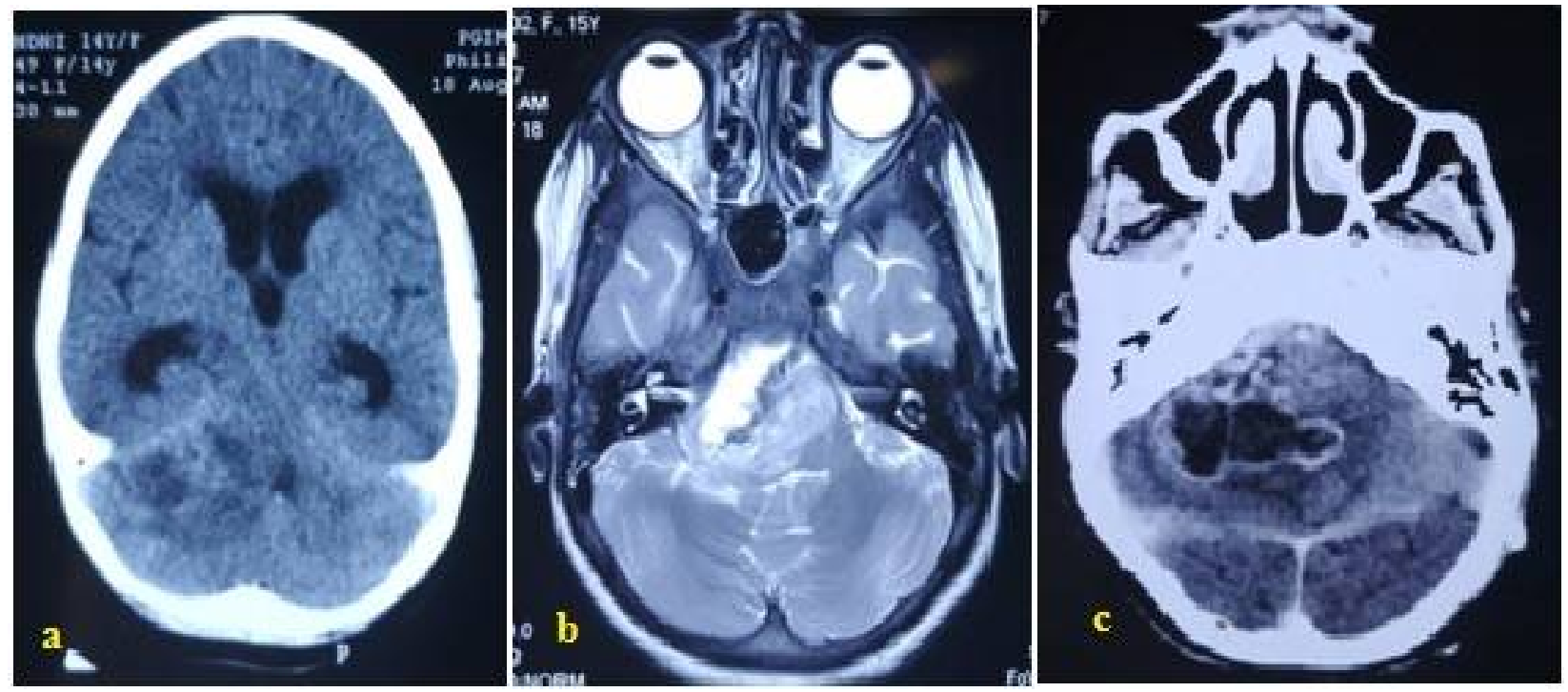

FIGURE 1. (a) Axial Noncontrast CT showing an iso to hypodense right CP angle mass lesion with obstructive hydrocephalous. (b) T2 weighted MRI image showing lesion heterogeneously hyperintense with a linear hypointensity. (c) Axial contrast CT image showing lesion enhancing heterogeneously with cystic component.

\section{DISCUSSION}

Most of the gliomas including GBM occur in the cerebral hemispheres, and posterior fossa glioma is uncommon, estimating as $1.5 \%$ in the cerebellum and $4.1 \%$ in the brainstem [2]. However primary GBM arising in the CPA is further rare with a few reported cases [1], [3]-[7] depending on the origin of the tumour primary CPA glioblastoma can be divided into two types. The first type of the CPA glioblastoma is an intraaxial tumour originated from brain stem or cerebellum with an exophytic growth into the CPA [4] -[6], [8]. This type of GBM is rare, and especially cerebellar GBM with the exophytic growth pattern is very rare, and up to date, four cases have been reported in the literature [3], [4], [8]. Two cases showed exophytic growth into CPA, and the other two cases were in the crural/quadrigeminal cistern and cisterna magna, respectively.

The second type is an extraaxial CPA glioblastoma. There has been one case of primary extraaxial GBM in the CPA, arising from the proximal portion of cranial nerve VIII [7]. Few possible mechanisms were documented regarding the origin of primary extraaxial GBM in the CPA. One is that the tumour arose from cranial nerve system tissue within proximal cranial nerve itself, and the other is that the tumour originated from heterotopic neuroglial cells in the leptomeninges covering the proximal cranial nerve or brainstem [1], [7]. 
In patients with cerebellopontine GBM the clinical features are similar to those of other aggressive fast growing infratentorial tumors. Signs and symptoms include headache, nausea, vomiting, and cerebellar dysfunction including ataxia, imbalance and unsteady gait, ipsilateral cranial nerves [9]-[11].

The radiological features of posterior fossa GBM are nonspecific [10]-[12]. Lesions occur laterally in the cerebellopontine angle. The lesions are typically infiltrating with indistinct margins. Signal characteristics are heterogenous, often with necrotic and cystic components. A thick and irregular wall is commonly seen. Irregular peripheral enhancement occurs following contrast administration. Edema is usually present and obstructive hydrocephalus is common.

Other features as histology and biology of cerebellar GBM are similar to that of cerebral GBM.11 This includes malignant tumour cells, mitoses, hypercellularity, pleomorphism and neoangiogenesis. The presence of necrosis helps differentiate GBM from anaplastic astrocytoma or from well-differentiated astrocytoma [9].

Cerebellar GBM has poor prognosis as with any GBM and any paediatric malignant brain tumour. This is attributed to rapid tumour progression, locally aggressive behaviour as well as the common findings of CSF pathway spread [12]. Early intervention has been advocated to increase the disease-free interval and to prolong survival includes, aggressive surgery as well as aggressive radiation and chemotherapy [9], [12]. Despite these measures; however, survival of children with CP angle GBM is very poor. However, the optimal management for the CPA glioblastoma is to be defined because of its rarity.

\section{CONCLUSIONS}

Primary glioblastoma arising in the CPA in paediatric age group although very rare and cannot always be differentiated from other usual benign tumours at this site radiologically, we should keep GBM in the differential diagnosis of an atypical lesion of the cerebellopontine angle, when patient presented with rapid clinical deterioration due to fast progression of the lesion.

\section{REFERENCES}

1. Arnautovic $\mathrm{Kl}$, Husain MM, Linskey ME. Cranial nerve root entry zone primary cerebellopontine angle gliomas: a rare and poorly recognized subset of extraparenchymal tumors. J Neurooncol. 2000; 49:205-212. [PubMed].

2. Larjavaara S, Mäntylä R, Salminen T, Haapasalo H, Raitanen J, Jääskeläinen J, et al. Incidence of gliomas by anatomic location. Neuro Oncol. 2007; 9:319-325. [PMC free article] [PubMed].

3. Kasliwal MK, Gupta DK, Mahapatra AK, Sharma MC. Multicentric cerebellopontine angle glioblastoma multiforme. Pediatr Neurosurg. 2008; 44:224-228. [PubMed].

4. Matsuda M, Onuma K, Satomi K, Nakai K, Yamamoto T, Matsumura A. Exophytic cerebellar glioblastoma in the cerebellopontine angle: case report and review of the literature. J Neurol Surg Rep. 2014;75: e67-e72. [PMC free article] [PubMed].

5. Salunke P, Sura S, Tewari MK, Gupta K, Khandelwal NK. An exophytic brain stem glioblastoma in an elderly presenting as a cerebellopontine angle syndrome. $\mathrm{Br} J$ Neurosurg. 2012; 26:96-98. [PubMed].

6. Swaroop GR, Whittle IR. Exophytic pontine glioblastoma mimicking acoustic neuroma. J Neurosurg Sci. 1997; 41:409-411. [PubMed].

7. Wu B, Liu W, Zhu H, Feng H, Liu J. Primary glioblastoma of the cerebellopontine angle in adults. J Neurosurg. 2011; 114:1288-1293. [PubMed].

8. Yamamoto $M$, Fukushima $T$, Sakamoto $S$, Tsugu $H$, Nagasaka S, Hirakawa K, et al. Cerebellar gliomas with exophytic growth--three case reports. Neurol Med Chir (Tokyo) 1997; 37:411-415. [PubMed].

9. Chin HW, Maruyama Y, Tibbs P. Cerebellar glioblastoma in childhood. J Neuro-Oncol. 1984; 2:79-84. [PubMed].

10. Kuroiwa T, NumaguchiY, Rothman MI, et al. Posterior Fossa Glioblastoma Multiforme MR Findings. Am J Neuroradiol. 1995; 16:583-9. [PubMed].

11. Luccarelli G. Glioblastoma multiforme of the cerebellum description of three cases. Acta Neurochir. 1980; 53:10716. [PubMed].

12. Katz DS, Poe LB, Winfield JA, et al. A rare case of cerebellar glioblastoma multiforme in childhood MR imaging. Clin Imag. 1995; 19:162-4. [PubMed]. 